\title{
Electron and Hole Capture Cross-Sections of Fe Acceptors in GaN:Fe Epitaxially Grown on Sapphire
}

\author{
T. AGGERSTAM,${ }^{1,2}$ A. PINOS,${ }^{1}$ S. MARCINKEVIČIUS,${ }^{1}$ M. LINNARSSON, ${ }^{1}$ \\ and S. LOURDUDOSS ${ }^{1}$ \\ 1.-Department of Microelectronics and Applied Physics, Royal Institute of Technology, Electrum \\ 229, 16440 Kista, Sweden. 2.—e-mail: aggis@imit.kth.se
}

Carrier trapping of $\mathrm{Fe}^{3+} / \mathrm{Fe}^{2+}$ deep acceptors in epitaxially grown $\mathrm{GaN}: \mathrm{Fe}$ on sapphire was studied by time-resolved photoluminescence. For the investigated Fe doping levels on the order of $10^{18} \mathrm{~cm}^{-3}$, the luminescence decay times are strongly dependent on the Fe concentration, indicating that Fe centers act as predominant nonradiative recombination channels. Linear dependence of the decay time on the iron concentration allows estimation of the electron capture cross-section for the $\mathrm{Fe}^{3+}$ ions, which is equal to $1.9 \times 10^{-15} \mathrm{~cm}^{2}$. The upper bound for the cross-section of the hole capture of $\mathrm{Fe}^{2+}$ was evaluated as $1 \times 10^{-15} \mathrm{~cm}^{2}$.

Key words: GaN, Fe, semi-insulating, high electron mobility transistor (HEMT), deep acceptor, electron capture cross section, metal-organic vapor phase epitaxy (MOVPE)

\section{INTRODUCTION}

$\mathrm{AlGaN} / \mathrm{GaN}$ high electron mobility transistors (HEMTs) are excellent candidates for high-power and high-frequency applications at elevated temperatures. For its performance to be brought out fully, a HEMT structure is grown on a semi-insulating (SI) layer or substrate to secure drain-source current saturation, channel pinch-off and low loss at high frequencies. Since SI GaN substrates are expensive, an epitaxial layer of an SI buffer layer on a relatively cheap sapphire substrate is normally used. ${ }^{1}$ A straightforward way of achieving semiinsulation is to compensate the residual donors by deep acceptors. Fe acts as a deep acceptor in $\mathrm{GaN}^{2,3}$ and it has been employed to dope buffer layers in AlGaN/GaN HEMT structures. ${ }^{4,5}$ The unintentionally doped buffer layer is otherwise $n$-type, which would result in parallel conduction in HEMT structures. Because of this technological importance, properties of $\mathrm{Fe}$ in $\mathrm{GaN}$ have been studied quite extensively. These studies, however, were mainly concentrated on Fe energy levels within the

(Received April 10, 2007; accepted June 15, 2007;

published online September 14, 2007) host material as well as intra-ion transitions. Properties of free carrier capture and recombination at the Fe centers, including capture cross-sections, have barely been approached. An electron capture cross-section of $2.3 \times 10^{-16} \mathrm{~cm}^{2}$ for Fe-related centers has been evaluated by Polyakov and co-workers in Fe-implanted and annealed layers by means of admittance spectroscopy. ${ }^{6}$ However, as the authors state in their subsequent publication, ${ }^{7}$ annealing does not completely eliminate implantation-induced defects; thus, this cross-section is attributed to $\mathrm{Fe}$ complexes with irradiation defects rather than to pure Fe centers. For a study of deep Fe acceptors, which are $\mathrm{Fe}^{3+}$ in the neutral state and $\mathrm{Fe}^{2+}$ in the excited state, doped samples are preferable. In this article, we estimate carrier capture cross-sections in Fe-doped GaN based on experimental time-resolved photoluminescence (PL) data.

\section{EXPERIMENTAL PROCEDURE}

The GaN films studied were grown by low-pressure metal-organic vapor phase epitaxy (LP-MOV$\mathrm{PE}$ ) on $c$-plane sapphire. Trimethylgallium (TMG) and ammonia were used as precursors. Ferrocene $\left(\mathrm{Cp}_{2} \mathrm{Fe}\right)$ was used as the iron source. A film was 
grown at $1,050^{\circ} \mathrm{C}$ and 200 mbar on a $100 \mathrm{~nm}$ thick $\mathrm{GaN}$ nucleation layer grown at $550^{\circ} \mathrm{C}$. Before the growth, the substrate was subjected to an in situ $1,200^{\circ} \mathrm{C}$ pretreatment. The $\mathrm{Fe}$ concentration in $\mathrm{GaN}: \mathrm{Fe}$ was varied with the ferrocene precursor flow, and its chemical content was determined by secondary ion mass spectrometry (SIMS) using a Cameca ims $4 \mathrm{f}$ instrument. A primary sputter beam of $8 \mathrm{keV}{ }^{16} \mathrm{O}_{2}^{+}$ions was applied, and secondary ions of ${ }^{56} \mathrm{Fe}^{+}$were detected. For depth profiling, the primary beam was rastered over an area of $200 \times 200 \mu \mathrm{m}^{2}$ and the signal was recorded from the central part of this area $(60 \mu \mathrm{m}$ in diameter $)$. In order to assess that $\mathrm{Fe}$ incorporation does not result in a drastic variation of the crystal quality, we recorded double crystal rocking curves for symmetric (002) and asymmetric (102) orientations, using Philips X'Pert MRD and a symmetric four-crystal monochromator in $\mathrm{Ge}(220)$ configuration. Fe-doped samples were compared with an undoped GaN layer grown under identical conditions. For this film, the residual electron concentration, as determined by Hall effect measurements, was $1 \times 10^{16} \mathrm{~cm}^{-3}$.

Carrier capture and recombination dynamics in GaN films were studied by time-resolved photoluminescence (PL) using third harmonic pulses ${ }^{8}$ at a $267 \mathrm{~nm}$ central wavelength of a mode-locked femtosecond Ti:sapphire laser (pulse repetition frequency $76 \mathrm{MHz}$ ). The average excitation intensity and the spot size diameter were $5 \mathrm{~mW}$ and $60 \mu \mathrm{m}$, respectively. Averaged over two absorption lengths and the spot size, the photoexcited carrier density was $2 \times 10^{17} \mathrm{~cm}^{-3}$. In this estimation, reflectivity and absorption coefficients, equal to 0.15 and $1.7 \times 10^{5} \mathrm{~cm}^{-1}$, respectively, ${ }^{9}$ were considered. The photoluminescence signal was detected with a synchroscan streak camera combined with a $0.25 \mathrm{~m}$ spectrometer. The temporal resolution of the system's response was 6 ps. Experiments were carried out at room temperature.

\section{RESULTS AND DISCUSSION}

Table I summarizes SIMS and X-ray diffraction measurement results for the studied GaN:Fe layers. The SIMS data present the total Fe dopant concentration. The full-width at half maximum (FWHM) values of the X-ray diffraction rocking curves show a minor influence of the Fe doping on the crystalline quality of the samples.
Figure 1 shows spectrally integrated PL transients for the GaN:Fe layers measured at the energy of the GaN band gap (3.5 eV). The signal decay is of a single exponential type, which suggests nonradiative recombination as the main carrier recombination channel. Comparison of the PL transients for the Fe-doped and the undoped samples allows us to conclude that this nonradiative recombination proceeds through the Fe centers, since, for the undoped sample (inset in Fig. 1), the PL decay is at least an order of magnitude slower than that for the Fedoped samples. Obviously, doped and undoped GaN layers contain numerous native carrier traps and recombination centers related to point and extended defects. However, their influence on carrier capture in the Fe-doped layers can be considered to be minor for the Fe concentrations used in this study. This assumption is based on the X-ray data and the relatively long recombination time in the undoped GaN layer. It is known that the broadening of the FWHM of the X-ray rocking curve in the (102) asymmetric orientation is associated with edge-type threading dislocations. ${ }^{10}$ From the little variation in FWHM of the X-ray rocking curves with increasing Fe concentration, see Table I, it is clear that the concentration of the trap-inducing dislocations should be relatively constant in all the samples. The latter observation suggests similar concentrations of the native traps and related carrier capture times in the Fe-doped and the undoped samples.

The PL decay times for all Fe-doped layers are gathered in Fig. 2. The decay times were extracted from the PL transients after deconvolution with the system response. The decay times are inversely proportional to the total $\mathrm{Fe}$ concentration. Observed dependence of the PL decay times on the Fe ion concentration allows us to evaluate cross-sections of the electron capture to the Fe centers. Here, we assume that all iron dopants are either in the $\mathrm{Fe}^{3+}$ or $\mathrm{Fe}^{2+}$ state. Residual donors, which would ionize neutral $\mathrm{Fe}^{3+}$ into excited $\mathrm{Fe}^{2+}$, are present at concentrations that are much lower than that of the Fe ions. Therefore, prior to the photoexcitation, the majority of $\mathrm{Fe}$ ions are in the neutral state, i.e., $\mathrm{Fe}^{3+}$, with only $1 \times 10^{16} \mathrm{~cm}^{-3}$ in the $\mathrm{Fe}^{2+}$ state. During the PL process, $\left[\mathrm{Fe}^{2+}\right]$ is a dynamic quantity because of the photoexcited electron and hole trapping (electron trapping changes $\mathrm{Fe}^{3+}$ to $\mathrm{Fe}^{2+}$; hole trapping changes it back). Nevertheless, the single exponential decay with time constant $\tau$ agrees with a linear model in

Table I. Total Iron Concentration Determined by Secondary Ion Mass Spectrometry and Full-Width at Half Maximum Values of the X-Ray Diffraction Rocking Curves for Symmetric (002) and Asymmetric (102) Orientations for the GaN:Fe Samples

\begin{tabular}{|c|c|c|c|c|c|c|}
\hline Sample & $\mathbf{a}$ & $\mathbf{b}$ & $\mathbf{c}$ & d & $\mathbf{e}$ & $\mathbf{f}$ \\
\hline $\mathrm{Fe}\left(\times 10^{18} \mathrm{~cm}^{-3}\right)$ & 1.7 & 2.4 & 3.8 & 5.0 & 7.0 & 10 \\
\hline FWHM from (002) (arcsec) & 301 & 298 & 285 & 360 & 360 & 357 \\
\hline FWHM from (102) (arcsec) & 452 & 420 & 478 & 456 & 486 & 456 \\
\hline
\end{tabular}




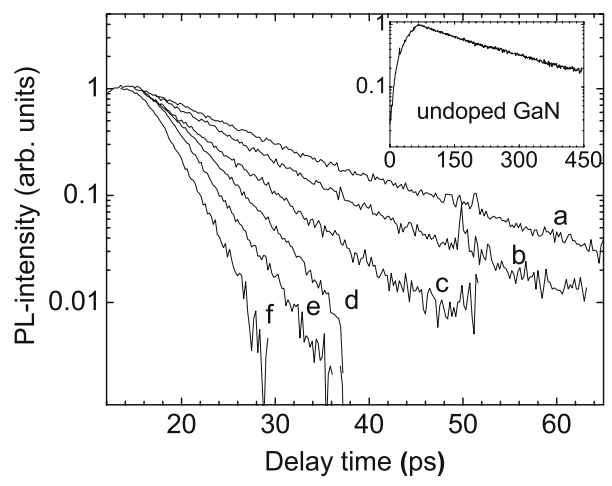

Fig. 1. Normalized PL transients for Fe-doped GaN with doping concentration increasing from sample a through $f$. The inset shows the corresponding data for the undoped sample.

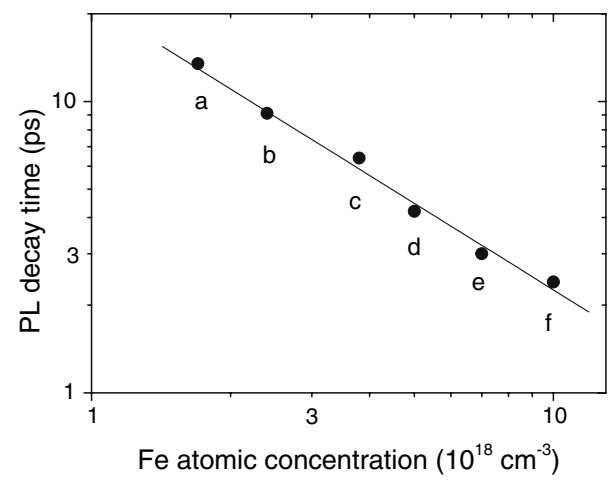

Fig. 2. PL decay times for samples with different Fe concentrations.

which a small fraction of the total Fe concentration is ionized during the recombination dynamics. In the opposite case, the PL decay would experience multiexponential behavior. If, accordingly, both $\mathrm{Fe}^{3+}$ or $\mathrm{Fe}^{2+}$ concentrations are considered constant during the recombination, the PL decay time $\tau$ and the center concentrations can be related as follows: ${ }^{11}$

$$
\frac{1}{\tau}=v_{\mathrm{e}} \sigma_{\mathrm{e}}\left[\mathrm{Fe}^{3+}\right]+v_{\mathrm{h}} \sigma_{\mathrm{h}}\left[\mathrm{Fe}^{2+}\right]
$$

where $\left[\mathrm{Fe}^{3+}\right]$ and $\left[\mathrm{Fe}^{2+}\right]$ denote concentrations of neutral and ionized Fe centers, $v_{\mathrm{e}(\mathrm{h})}$ and $\sigma_{\mathrm{e}(\mathrm{h})}$ are the electron and hole thermal velocity ${ }^{12} v_{\mathrm{e}(\mathrm{h})}=$ $\sqrt{8 k T /\left(\pi m_{\mathrm{e}(\mathrm{h})}^{*}\right)}$ and capture cross-section, respectively. The values of $v_{\mathrm{e}}$ and $v_{\mathrm{h}}$ used are $2.4 \times 10^{7} \mathrm{~cm} /$ $\mathrm{s}$ and $1.2 \times 10^{7} \mathrm{~cm} / \mathrm{s}$ and are obtained with values of electron and hole effective mass equal to $m_{\mathrm{e}}{ }^{*}=$ $0.20 m_{0}$ and $m_{\mathrm{h}}{ }^{*}=0.80 m_{0}$, respectively. The total iron concentration measured by SIMS is $[\mathrm{Fe}]=[$ $\left.\mathrm{Fe}^{3+}\right]+\left[\mathrm{Fe}^{2+}\right]$. One can rewrite Eq. 1 as:

$$
\frac{1}{\tau}=v_{\mathrm{e}} \sigma_{\mathrm{e}}[\mathrm{Fe}]+\left(v_{\mathrm{h}} \sigma_{\mathrm{h}}-v_{\mathrm{e}} \sigma_{\mathrm{e}}\right)\left[\mathrm{Fe}^{2+}\right]
$$

According to this model, by plotting $1 / \tau$ versus $[\mathrm{Fe}]$, one should obtain a straight line, which, indeed, is the case, see Fig. 2. This also confirms that $\mathrm{Fe}^{3+} / \mathrm{Fe}^{2+}$ is the predominant nonradiative recombination channel. The slope linear dependence allows one to evaluate $\sigma_{\mathrm{e}}$ for $\mathrm{Fe}^{3+}$ as $1.9 \times 10^{-15} \mathrm{~cm}^{2}$. We evaluated the hole capture cross-section be simulating PL transients with a numerical model ${ }^{13}$ that took into account radiative and nonradiative carrier recombination as well as the measurement system's temporal resolution. In the simulations, the concentration of $\mathrm{Fe}^{2+}$ and $\mathrm{Fe}^{3+}$ states was treated dynamically; trapping of $\mathrm{Fe}$ and native defects was considered. Figure 3 shows simulation results for the sample (a) with $1.7 \times 10^{18} \mathrm{~cm}^{-3} \mathrm{Fe}$ concentration performed for the electron capture cross-section $\sigma_{\mathrm{e}}=1.9 \times 10^{-15} \mathrm{~cm}^{2}$ and different hole capture cross-sections. As can be seen, successful fitting of calculated and experimental values requires a hole capture cross-section of $\sigma_{\mathrm{h}}=1 \times 10^{15} \mathrm{~cm}^{2}$. With larger cross-sections, the calculated PL decay is too fast compared to the experimental data. For $\sigma_{\mathrm{h}}<1 \times$ $10^{15} \mathrm{~cm}^{2}$, the variation of hole capture cross-section does not have a major influence on the shape of the PL transient. Thus, $\sigma_{\mathrm{h}}=1 \times 10^{15} \mathrm{~cm}^{2}$ can be assumed as the higher limit of the hole capture crosssection for $\mathrm{Fe}^{2+}$ in GaN. The hole capture-cross section seems to assume a lower value than the corresponding parameter for the electrons. This is in accord with the multiphonon capture mechanism valid for the $\mathrm{Fe}$ recombination centers ${ }^{14}$ and the $\mathrm{Fe}^{3+/ 2+}$ level position in GaN. PL excitation measurements show that the acceptor level is situated in the band gap at $2.5 \mathrm{eV}$ from the valence band, with an excited level at $3 \mathrm{eV}$ from the valence band. ${ }^{15}$ This means that energetically $\mathrm{Fe}^{3+/ 2+}$ levels are closer to the conduction than to the valence band. In the multiphonon capture model, $\sigma \propto \exp \left(-E_{t} / k T\right)$, where $E_{t}$ is the trap binding energy. ${ }^{12}$ Clearly, with the Fe centers being closer to the conduction band, electron trapping is favored.

\section{CONCLUSION}

In conclusion, carrier trapping due to $\mathrm{Fe}^{3+} / \mathrm{Fe}^{2+}$ in epitaxially grown GaN:Fe was studied by

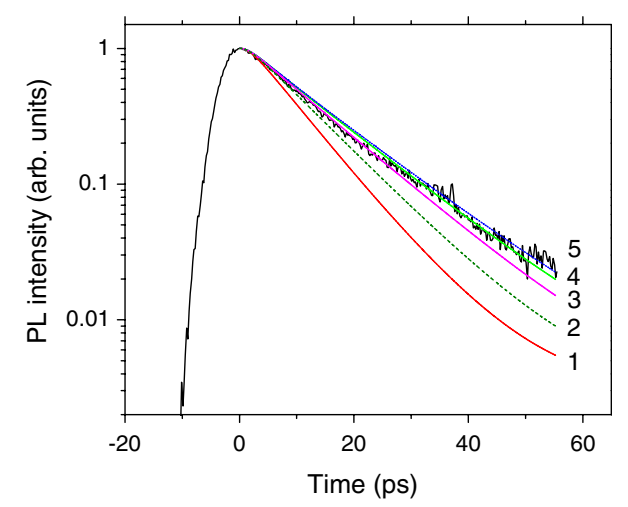

Fig. 3. Experimental and simulated $\mathrm{PL}$ transients for sample a $(\mathrm{Fe}=$ $\left.1.7 \times 10^{18} \mathrm{~cm}^{-3}\right) ; \sigma_{\mathrm{e}}=1.9 \times 10^{-15} \mathrm{~cm}^{2}, \sigma_{\mathrm{h}}$ is equal to $3 \times 10^{-14}(1)$, $1 \times 10^{-14}(2), 3 \times 10^{-15}(3), 1 \times 10^{-15}(4)$ and $3 \times 10^{-16} \mathrm{~cm}^{2}(5)$. 
time-resolved photoluminescence. For the investigated $\mathrm{Fe}$ doping levels of the order of $10^{18} \mathrm{~cm}^{-3}$, the luminescence decay times were strongly dependent on the Fe concentration, indicating that Fe centers act as predominant nonradiative recombination channels. Inverse linear dependence of the decay time on the iron concentration allows one to estimate carrier capture cross-sections for the $\mathrm{Fe}$ ions. The electron capture cross-sections for $\mathrm{Fe}^{3+}$ ions was evaluated as $1.9 \times 10^{-15} \mathrm{~cm}^{2}$. For the hole capture for $\mathrm{Fe}^{2+}$, the upper cross-section limit of $1 \times 10^{-15}$ $\mathrm{cm}^{2}$ was derived.

\section{ACKNOWLEDGEMENTS}

Financial support from the Swedish Foundation for International Cooperation in Research and Higher Education (STINT), the Swedish Foundation for Strategic Research (SSF), and the Göran Gustafsson Foundation is gratefully acknowledged.

\section{REFERENCES}

1. S.M. Hubbard, G. Zhao, D. Pavlidis, W. Sutton, and E. Cho, J. Cryst. Growth 284, 297 (2005).

2. J. Baur, K. Maier, M. Kunzer, U. Kaufmann, J. Schneider, H. Amano, I. Akasaki, T. Detchprohm, and K. Hiramatsu, Appl. Phys. Lett. 64, 857 (1994).
3. S. Heikman, S. Keller, S.P. DenBaars, and U.K. Mishra, Appl. Phys. Lett. 81, 439 (2002).

4. Z. Bougrioua, M. Azize, A. Jimenez, A.-F. Braña, P. Lorenzini, B. Beaumont, E. Muñoz, and P. Gibart, Phys. Status Solidi C 2, 2424 (2005).

5. T. Aggerstam, M. Sjödin, and S. Lourdudoss, Phys. Status Solidi C 3, 2373 (2006).

6. A.Y. Polyakov, N.B. Smirnov, A.V. Govorkov, N.V. Pashkova, A.A. Shlensky, S.J. Pearton, M.E. Overberg, C.R. Abernathy, J.M. Zavada, and R.G. Wilson, J. Appl. Phys. 93, 5388 (2003).

7. A.Y. Polyakov, N.B. Smirnov, A.V. Govorkov, S.J. Pearton, and J.M. Zavada, J. Phys.: Condens. Matter 16, 2967 (2004).

8. F. Rotermund and F. Petrov, Opt. Lett. 23, 1040 (1998).

9. J.F. Muth, J.H. Lee, I.K. Shmagin, R.M. Kolbas, H.C. Casey Jr., B.P. Keller, U.K. Mishra, and S.P. DenBaars, Appl. Phys. Lett. 71, 2572 (1997).

10. J. I. Pankove, S. Bloom, and G. Harbeke, RCA Rev. 36, 163 (1975).

11. D. Söderström, S. Marcinkevičius, S. Karlsson, and S. Lourdudoss, Appl. Phys. Lett. 70, 3374 (1997).

12. V.N. Abakumov, V.I. Perel, and I.N. Yassievich, Nonradiative recombination in semiconductors (The Netherlands: Amsterdam, 1991), p. 120.

13. A. Gaarder, S. Marcinkevičius, E. Rodríguez Messmer, and S. Lourdudoss, J. Cryst. Growth 226, 451 (2001).

14. A. Dadgar, R. Engelhardt, M. Kuttler, and D. Bimberg, Phys. Rev. B: Condens. Matter Mater. Phys. 56, 10241 (1997).

15. J. Baur, K. Maier, M. Kunzer, U. Kaufmann, and J. Schneider, Appl. Phys. Lett. 65, 2211 (1994). 